\title{
The Dynamic Beta Effect in CESR David Sagan
}

\section{Introduction}

In a colliding beam storage ring the Twiss parameters are affected by the quadrupolar focusing of the beam-beam interaction. Like any quadrupole error this 'dynamic beta' effect is enhanced by running near a half-integer or integer resonance. The recent change in the operating point of CESR so that the horizontal tune is just above a half-integer resonance has resulted in the dynamic beta effect having a noticeable effect on CESR operation. Under current colliding beam conditions the resulting change in horizontal beta has exceeded $\Delta \beta_{x} / \beta_{x}=0.5$.

In order to keep the notation as simple as possible while minimizing possible confusion, the following conventions have been adopted:

- Unless explicitly noted otherwise all Twiss parameters and beam sizes refer to the IP.

- A subscript of ' 0 ' denotes quantities calculated without the beam-beam interaction. For example, $\beta_{0}$ refers to the 'unperturbed' beta.

- Since the electron and positron bunch currents or sizes can be different, their effect on one another will not be symmetric (although in practice this asymmetry is usually small). Through the beam-beam interaction, this will result in different Twiss parameters and different beam sizes for each beam. '+' and '-' subscripts will be used to refer to the positron and electron beams respectively.

- 'Repeating' subscripts will be dropped from equations. For example, if Eq. (1) is applied to the horizontal plane for positrons, then all the variables get the subscript ' $x+$ '. Notice that without the beam-beam interaction it is assumed that the electrons and positrons have identical properties so that, for example, $\beta_{x 0} \equiv \beta_{x 0+} \equiv \beta_{x 0-}$. 


\section{Analysis}

Following Chao[1] the dynamic beta effect can be analyzed by writing the 1 -turn transfer matrix from IP to IP as

$$
\left(\begin{array}{cc}
\cos \mu & \beta \sin \mu \\
-\frac{1}{\beta} \sin \mu & \cos \mu
\end{array}\right)=\left(\begin{array}{cc}
1 & 0 \\
-\frac{1}{2 f} & 1
\end{array}\right)\left(\begin{array}{cc}
\cos \mu_{0} & \beta_{0} \sin \mu_{0} \\
-\frac{1}{\beta_{0}} \sin \mu_{0} & \cos \mu_{0}
\end{array}\right)\left(\begin{array}{cc}
1 & 0 \\
-\frac{1}{2 f} & 1
\end{array}\right),
$$

where the beam-beam interaction strength of $1 / f$ is given by

$$
\frac{1}{f_{x+}}=\frac{2 N_{-} r_{e}}{\gamma \sigma_{x-}\left(\sigma_{x-}+\sigma_{y-}\right)}
$$

with analogous formulas for $f_{x-}, f_{y+}$, and $f_{y-}$. The beam-beam parameter $\xi$ is defined by

$$
\xi \equiv \frac{\beta_{0}}{4 \pi f}
$$

$\xi$ is just the focusing strength of one beam on the other normalized by $\beta_{0}$. It is sometimes convenient to define another beam-beam parameter $\kappa$ by

$$
\kappa \equiv \frac{\beta}{4 \pi f}=\frac{\beta}{\beta_{0}} \xi
$$

Combining Eqs. (1), (2), (3), and (4) gives

$$
\begin{aligned}
\cos \mu & =\cos \mu_{0}-2 \pi \xi \sin \mu_{0} \\
& =\cos \mu_{0}-2 \pi \kappa \frac{\beta_{0}}{\beta} \sin \mu_{0}, \\
\sin \mu & =\frac{\beta_{0}}{\beta} \sin \mu_{0} .
\end{aligned}
$$

Eliminating $\mu$ from Eqs. (5) and (7) gives

$$
\frac{\beta}{\beta_{0}}=\left(1-(2 \pi \xi)^{2}+2(2 \pi \xi) \cot \mu_{0}\right)^{-1 / 2}
$$

Alternatively, in terms of $\kappa$, one finds

$$
\frac{\beta}{\beta_{0}}=\sqrt{1+\frac{(2 \pi \kappa)^{2}}{\sin ^{2} \mu_{0}}}-(2 \pi \kappa) \cot \mu_{0} .
$$

Figure 1 shows $\beta / \beta_{0}$ as calculated from Eq. (8) as a function of $Q_{0} \equiv \mu_{0} / 2 \pi$ for three different values of $\xi$. As can be seen from the figure, the dynamic beta effect is enhanced (large deviation of $\beta / \beta_{0}$ from 1 ) when $\mu_{0}$ is near an integer or halfinteger as expected. Furthermore, from the figure, it is seen that for tunes just above 


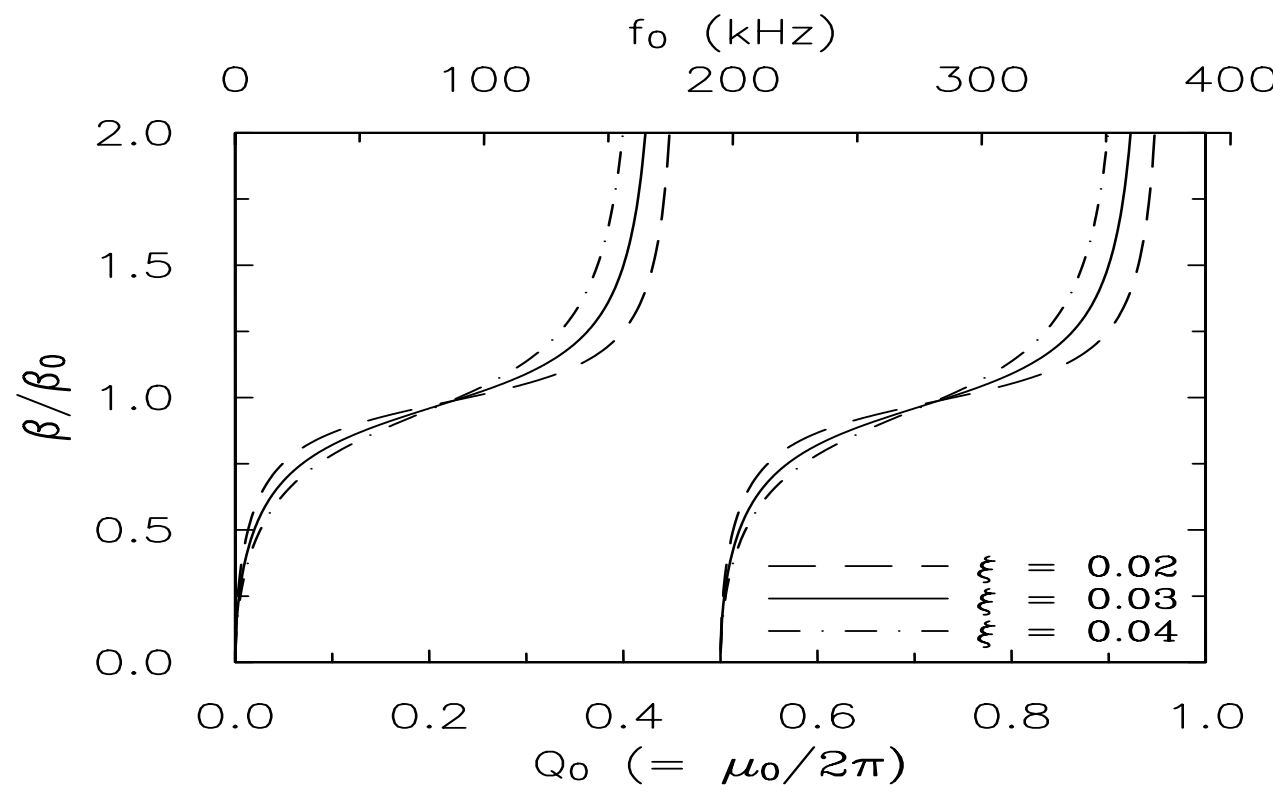

Figure 1: $\beta$ relative to $\beta_{0}$ as a function of tune for three different values of $\xi$. The top scale shows the tune in $\mathrm{kHz}$.

a integer or half-integer resonance the dynamic beta effect causes a reduction in $\beta$. This, of course, is what is desired for increased luminosity. As an example, the current operating point has a design horizontal tune of $Q_{x 0}=10.52$ (204 kHzfractional tune). Under the assumption that $\xi$ is in the vicinity of 0.03 (see below), this implies that there is a large reduction in beta of $\beta_{x} / \beta_{x 0} \sim 0.5$. Additionally, with the present vertical tune of $Q_{y 0}=9.60$ (233 $\mathrm{kHzfractional}$ tune), the reduction in vertical beta is $\beta_{y} / \beta_{y 0} \sim 0.8$

Along with the change in $\beta$ at the IP there will also be a beta-wave throughout the ring. The calculation of the beta-wave is straightforward. Given the unperturbed Twiss parameters at some point $s$ in the ring, the unperturbed transfer matrix from IP to $s$ is $[2]$

$$
\begin{aligned}
\mathbf{M}_{0}(s \mid \mathrm{IP}) & =\left(\begin{array}{ll}
m_{0,11} & m_{0,12} \\
m_{0,21} & m_{0,22}
\end{array}\right) \\
& =\left(\begin{array}{cc}
\sqrt{\frac{\beta_{0}(s)}{\beta_{0}(I P)}} \cos \phi_{0}(s) & \sqrt{\beta_{0}(s) \beta_{0}(I P)} \sin \phi_{0}(s) \\
\frac{-\left(\sin \phi_{0}(s)+\alpha_{0}(s) \cos \phi_{0}(s)\right)}{\sqrt{\beta_{0}(s) \beta_{0}(I P)}} & \sqrt{\frac{\beta_{0}(I P)}{\beta_{0}(s)}}\left(\cos \phi_{0}(s)-\alpha_{0}(s) \sin \phi_{0}(s)\right)
\end{array}\right),
\end{aligned}
$$

where $\phi_{0}(s)$ is the unperturbed phase advance from the IP to point $s$ and the fact that $\alpha_{0}(\mathrm{IP})=0$ has been used. Given any transfer matrix $\mathbf{M}$, the transfer matrix for 
the Twiss parameters is $[2]$

$$
\left(\begin{array}{c}
\beta\left(s_{2}\right) \\
\alpha\left(s_{2}\right) \\
\gamma\left(s_{2}\right)
\end{array}\right)=\left(\begin{array}{ccc}
m_{11}^{2} & -2 m_{11} m_{12} & m_{12}^{2} \\
-m_{11} m_{21} & 1+2 m_{12} m_{21} & -m_{12} m_{22} \\
m_{21}^{2} & -2 m_{22} m_{21} & m_{22}^{2}
\end{array}\right)\left(\begin{array}{c}
\beta\left(s_{1}\right) \\
\alpha\left(s_{1}\right) \\
\gamma\left(s_{1}\right)
\end{array}\right)
$$

Using the beam-beam kick matrix from Eq. (1) along with Eqs. (10), and (11) the beta function with the beam-beam interaction is

$$
\beta(s)=\beta(\mathrm{IP})\left(m_{0,11}-m_{0,12} \frac{2 \pi \xi}{\beta_{0}(\mathrm{IP})}\right)^{2}+\frac{m_{0,12}^{2}}{\beta(\mathrm{IP})} .
$$

If the beam-beam interaction is small enough one can use first order perturbation theory (cf. Sands[3] Eq. 2.105) to obtain more simply

$$
\frac{\Delta \beta(s)}{\beta_{0}(s)}=\frac{\beta_{0}(\mathrm{IP})}{2 f} \frac{\cos \left(2 \phi_{0}(s)-\mu_{0}\right)}{\sin \mu_{0}} .
$$

Evaluating Eq. (13) at the IP gives

$$
\frac{\Delta \beta(\mathrm{IP})}{\beta_{0}(\mathrm{IP})}=\frac{\beta_{0}(\mathrm{IP})}{2 f} \frac{\cos \mu_{0}}{\sin \mu_{0}} .
$$

Combining Eqs. (13) and (14) then gives

$$
\frac{\Delta \beta(s)}{\beta_{0}(s)}=\frac{\Delta \beta(\mathrm{IP})}{\beta_{0}(\mathrm{IP})} \cdot \frac{\cos \left(2 \phi_{0}(s)-\mu_{0}\right)}{\cos \mu_{0}} .
$$

As an example, with the present horizontal tune of $Q_{x 0}=10.52$, the horizontal beta-wave is roughly given by

$$
\frac{\Delta \beta_{x}(s)}{\beta_{0 x}(s)} \approx-0.5 \cos \left(2 \phi_{x 0}(s)-\mu_{x 0}\right) .
$$

One consequence of this beta-wave is that it changes the emittance function $\mathcal{H}_{x}(s)$ (Sands[3] Eq. 5.71)

$$
\mathcal{H}_{x}(s)=\frac{1}{\beta_{x}}\left\{\eta_{x}^{2}+\left(\beta_{x} \eta^{\prime}-\frac{1}{2} \beta_{x}^{\prime} \eta_{x}\right)^{2}\right\},
$$

and this will affect the horizontal emittance (see below).

With the present vertical tune of $Q_{y 0}=9.60$, the vertical beta-wave is not as large as the horizontal and can be approximated by

$$
\frac{\Delta \beta_{y}(s)}{\beta_{0 y}(s)} \approx-0.2 \cos \left(2 \phi_{y 0}(s)-\mu_{y 0}\right)
$$




\section{Synch Light Luminosity Calculation}

One result of the dynamic beta effect is that it throws off the luminosity as calculated from the vertical beam heights observed at the electron and positron synch light monitors. As will be shown, this can be used to verify the presence of the dynamic beta effect. The luminosity is calculated from the vertical beam size by using the formula

$$
\mathcal{L}=\frac{f_{\text {rev }}}{4 \pi \bar{\sigma}_{x} \bar{\sigma}_{y}} \sum_{i=1}^{n_{\text {bunch }}} N_{i+} N_{i-}
$$

where $f_{\text {rev }}$ is the revolution frequency, $n_{\text {bunch }}$ is the number of bunches, and $N_{i-}$ and $N_{i+}$ are the number of positrons and electrons respectively in the $i^{\text {th }}$ bunch. To take into account the fact that the beam sigmas will be different, $\bar{\sigma}_{x}$ and $\bar{\sigma}_{y}$ in Eq. (19) are defined by the overlap integral between the two beams. For example, $\bar{\sigma}_{x}$ is defined by

$$
\frac{1}{2 \pi \bar{\sigma}_{x}^{2}} \int_{-\infty}^{\infty} d x e^{-x^{2} / 2 \bar{\sigma}_{x}^{2}} \cdot e^{-x^{2} / 2 \bar{\sigma}_{x}^{2}} \equiv \frac{1}{2 \pi \sigma_{x+} \sigma_{x-}} \int_{-\infty}^{\infty} d x e^{-x^{2} / 2 \sigma_{x+}^{2}} \cdot e^{-x^{2} / 2 \sigma_{x-}^{2}} .
$$

Eq. (20) is easily integrated. The general result for either the horizontal or vertical planes is

$$
\bar{\sigma}=\sqrt{\frac{\left(\sigma_{+}^{2}+\sigma_{-}^{2}\right)}{2}} .
$$

The beam sigmas $\sigma_{x+}, \sigma_{x-}, \sigma_{y+}$, and $\sigma_{y-}$ are calculated from the equations

$$
\sigma_{x}=\sqrt{\epsilon_{x} \beta_{x}}
$$

and

$$
\sigma_{y}=\sigma_{y}(l s) \cdot \sqrt{\frac{\beta_{y}(\mathrm{IP})}{\beta_{y}(l s)}},
$$

where $(l s)$ stands for the light source point. If the dynamic dynamic beta effect is ignored then the unperturbed values for $\epsilon_{x}, \beta_{x}$, and $\beta_{y}$ are used in Eqs. (22) and (23). Taking the dynamic beta effect into account complicates the calculation since a closed formula does not exist for the $\sigma_{x}$ or the $\sigma_{y}$. Fortunately, as outlined below, a relatively simple iterative method is effective for obtaining the beam sigmas.

Since the synch light monitors only gives beam heights averaged over all the bunches, the dynamic beta calculation outlined below calculates a single value for the beam sigmas based on the average electron and average positron bunch currents. With dynamic beta the beam size calculation starts with the horizontal plane. The procedure is as follows:

Step 0: Initial values are chosen (guessed at) for the $\sigma_{x}$ and the $\sigma_{y}$, and the dependence of $\epsilon_{x}$ as a function of $\xi_{x}$ is calculated using the detailed lattice for the ring. 
Step 1: Calculate $\xi_{x+}$ and $\xi_{x-}$ from Eqs. (2) and (3).

Step 2: Calculate $\beta_{x+}$ and $\beta_{x-}$ from Eq. (8).

Step 3: Calculate $\epsilon_{x+}$ and $\epsilon_{x-}$ from the curve of $\epsilon_{x}$ verses $\xi_{x}$.

Step 4: Calculate $\sigma_{x+}$ and $\sigma_{x-}$ from Eq. (22).

Step 5: If the $\sigma_{x}$ have not changed by more than $0.1 \%$ then continue with step 6. Otherwise loop back to step 1.

Step 6: Calculate $\bar{\sigma}_{x}$ from Eq. (21).

Notice that only an inexact value for $\sigma_{y}$ is used by the horizontal calculation. This is acceptable since the horizontal dynamic beta is only weakly affected by errors in $\sigma_{y}$. After the horizontal calculation the vertical calculation proceeds as follows:

Step 1: Calculate $\xi_{y+}$ and $\xi_{y-}$ from Eqs. (2) and (3).

Step 2: Calculate $\beta_{y+}$ and $\beta_{y-}$ from Eq. (8).

Step 3: Calculate $\beta_{y}(l s+)$ and $\beta_{y}(l s-)$ from Eq. (12)

Step 4: Calculate $\sigma_{y+}$ and $\sigma_{y-}$ from Eq. (23).

Step 5: If the $\sigma_{y}$ have not changed by more than $0.1 \%$ then continue with step 6 . Otherwise loop back to step 1 .

Step 6: Calculate $\overline{\sigma_{y}}$ from Eq. (21).

Since $\beta$ is only weakly dependent upon $\xi$ (cf. figure 1) the convergence for both the horizontal and vertical calculations is quite rapid and usually takes only a few iterations. Note that while $\xi$ has been used in the above procedure, with minor modifications $\kappa$ could have just as easily been used instead. One minor detail: In the results presented below the values for $N_{i+}$ and $N_{i-}$ as obtained from the beam button monitors have been scaled by an overall factor in order that their sum agrees with the CERN current monitor.

Figures 2 through 8 show the results of a dynamic beta calculation using data from two days of HEP running. In order to minimize possible abnormalities in the data the criterion for selecting the days for analysis was that there was relatively good luminosity on the day chosen and on the days just previous to the chosen day (e.g. days just after a start-up were avoided). The first day chosen was April 14, 1994 (Julian day 104). The lattice used on this date was C9A18A000.GE92S_4S. The second day chosen was August 1, 1994 (Julian day 213). The lattice used on this date was N9A18A600.FD92S_4S. The relevant parameters for both lattices are given in tables 1 and 2. The C9A18A000.GE92S_4S lattice has relatively high fractional tunes compared to the N9A18A600.FD92S_4S lattice and thus would not be expected to be affected as much by dynamic beta. For each day the luminosity and related parameters were calculated every 5 minutes and in the figures the parameters are plotted as 
Table 1: C9A18A000.GE92S_4S

\begin{tabular}{|c|l|}
\hline Parameter & Value \\
\hline$Q_{x 0}$ & $10.5736(225 \mathrm{kHz}$ frac. tune $)$ \\
\hline$Q_{y 0}$ & $9.6302(246 \mathrm{kHz}$ frac. tune $)$ \\
\hline$\beta_{x 0}$ & $1.0023 \mathrm{~m}$ \\
\hline$\beta_{y 0}$ & $0.0179 \mathrm{~m}$ \\
\hline$\epsilon_{x 0}$ & $0.226 \mu \mathrm{m} \cdot \mathrm{rad}$ \\
\hline$\sigma_{x 0}$ & $476 \mu \mathrm{m}$ \\
\hline$\gamma$ & $1.035 \cdot 10^{4}$ \\
\hline$N_{\text {bunches }}$ & 7 \\
\hline$f_{\text {rev }}$ & $2.56 \mu \mathrm{s}$ \\
\hline
\end{tabular}

Table 2: N9A18A600.FD92S_4S

\begin{tabular}{|c|l|}
\hline Parameter & Value \\
\hline$Q_{x 0}$ & $10.5235(204.2 \mathrm{kHz}$ frac. tune $)$ \\
\hline$Q_{y 0}$ & $9.5971(233 \mathrm{kHz}$ frac. tune $)$ \\
\hline$\beta_{x 0}$ & $1.0865 \mathrm{~m}$ \\
\hline$\beta_{y 0}$ & $0.0182 \mathrm{~m}$ \\
\hline$\epsilon_{x 0}$ & $0.200 \mu \mathrm{m} \cdot \mathrm{rad}$ \\
\hline$\sigma_{x 0}$ & $467 \mu \mathrm{m}$ \\
\hline$\gamma$ & $1.035 \cdot 10^{4}$ \\
\hline$N_{\text {bunches }}$ & 7 \\
\hline$f_{\text {rev }}$ & $2.56 \mu \mathrm{s}$ \\
\hline
\end{tabular}

a function of the total positron plus electron current. Figure 2 shows the luminosity as a function of total current. The three sets of data shown correspond to: A) Data from the CLEO detector which (presumably) gives the correct luminosity, B) The luminosity as calculated from the synch light monitors neglecting the dynamic beta effect, and C) The luminosity as calculated from the synch light monitors including the dynamic beta effect. In each graph there is a noticeable difference between neglecting and not neglecting the dynamic beta effect. For the C9A18A000.GE92S_4S run the three curves are too close together (i.e. the magnitude of the dynamic beta effect is too small) to make any conclusions. For the N9A18A600.FD92S_4S run, however, it is clear that one must take the dynamic beta effect into account. For the N9A18A600.FD92S_4S run the remaining difference between the CLEO luminosity and the calculation with the dynamic beta effect can be attributed to a number of factors: (1) The actual tunes will differ somewhat from the design tunes but it is the design tunes that are used in the calculation. (2) The hourglass effect $[6]$ will reduce 
the actual luminosity relative to the calculated value. (3) Any vertical dispersion at the synch light ports makes the vertical betatron size seem bigger than actual and this increases the actual luminosity relative to the calculated. (4) Finally, there is always the possibility of a resonant blowup in $\epsilon_{x}$ due to the non-linear part of the beam-beam interaction.

Figure 3 shows the average beta $\langle\beta\rangle \equiv\left(\beta_{+}+\beta_{-}\right) / 2$ normalized by the unperturbed beta as a function of total current. Since the horizontal tune is always closer to the half-integer resonance the reduction in $\left\langle\beta_{x}\right\rangle$ is always larger than $\left\langle\beta_{y}\right\rangle$. For the N9A18A600.FD92S_4S run the reduction in $\left\langle\beta_{x}\right\rangle$ is quite dramatic, being over a factor of 2 at the larger currents. This is opposite of what happens to the average horizontal emittance $\left\langle\epsilon_{x}\right\rangle \equiv\left(\epsilon_{x+}+\epsilon_{x-}\right) / 2$ as shown in Figure 4. For both lattices $\left\langle\epsilon_{x}\right\rangle$ is an increasing function of $\xi_{x}$ as shown in figure 5 . The result is that the drop in $\beta_{x}$ is partially offset by the rise in $\epsilon_{x}$ producing a $\sigma_{x}$ with only a weak current dependence as shown in figure 6 . [Note that for figure $6 \sigma_{y 0}$ is taken to be the sigma as calculated from Eq. (23) using the unperturbed beta. This is not the same as the sigma that one expects without the beam-beam interaction.]

The difference between $\xi, \kappa$, and $\Delta Q \equiv\left(\mu-\mu_{0}\right) / 2 \pi$ is shown in figures 7 and 8 as a function of total current. For the C9A18A000.GE92S_4S run the tunes are far enough away from the half-integer resonance so that the dynamic beta effect is small and $\kappa \approx \xi \approx \Delta Q$. On the other hand, for the N9A18A600.FD92S_4S run, $\kappa_{x}$ is clearly closer to $\Delta Q_{x}$ than $\xi_{x}$. Notice that for the N9A18A600.FD92S_4S run $\xi_{x}$ shows no signs of saturation and reaches a level of 0.05 at the highest current levels. $\Delta Q_{x}$ and $\kappa_{x}$, on the other hand, show some signs of saturation at the highest current levels and stay within the 'normal range' of less than 0.03 or so. This is in accord with the standard argument[4] that since a measure of the strength of the beam-beam force is the shift of the tune $\Delta Q$, and since the beam-beam induced resonances limit the performance of the machine, that for $\Delta Q$ there is a 'saturation limit' above which one cannot go without the beams falling out or the beams blowing up (which lowers $\Delta Q$ back to its limiting value). That $\kappa$ is a better approximation to $\Delta Q$ than $\xi$ is a general feature as shown in figure 9. In figure 9, Eqs. (4), (5), and (8) have been used to graph $\xi, \kappa$, and $\Delta Q$ as functions of $Q_{0}$ with $\xi$ somewhat arbitrarily fixed at a value of 0.04 . Over the range of $Q_{0}$ from 0.5 to 0.6 , which is the range where one would like to operate to take advantage of the dynamic beta effect, $\kappa$ is clearly closer to $\Delta Q$ than $\xi$. 

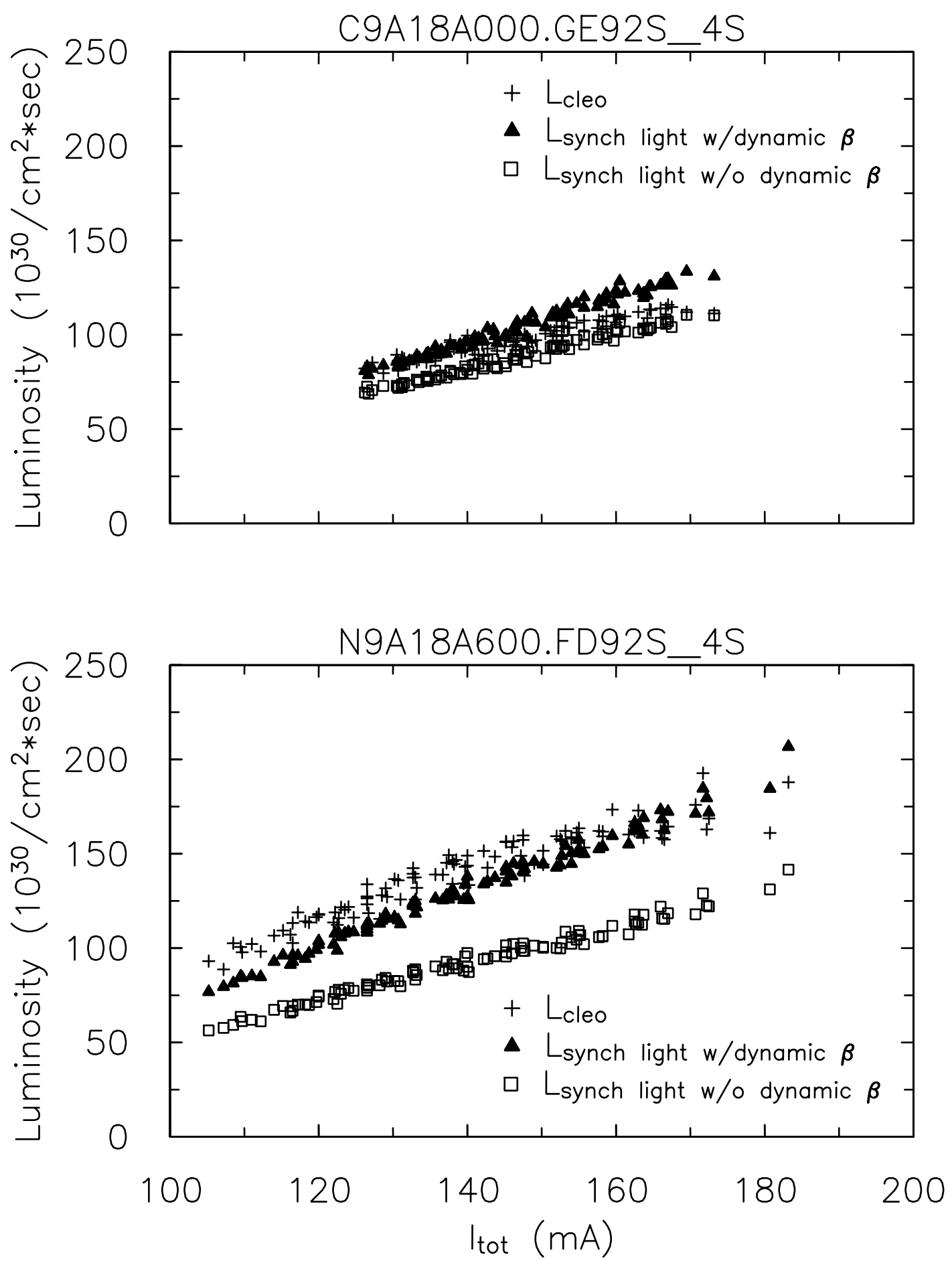

Figure 2: Luminocity as a function of total current for two days of HEP running. 

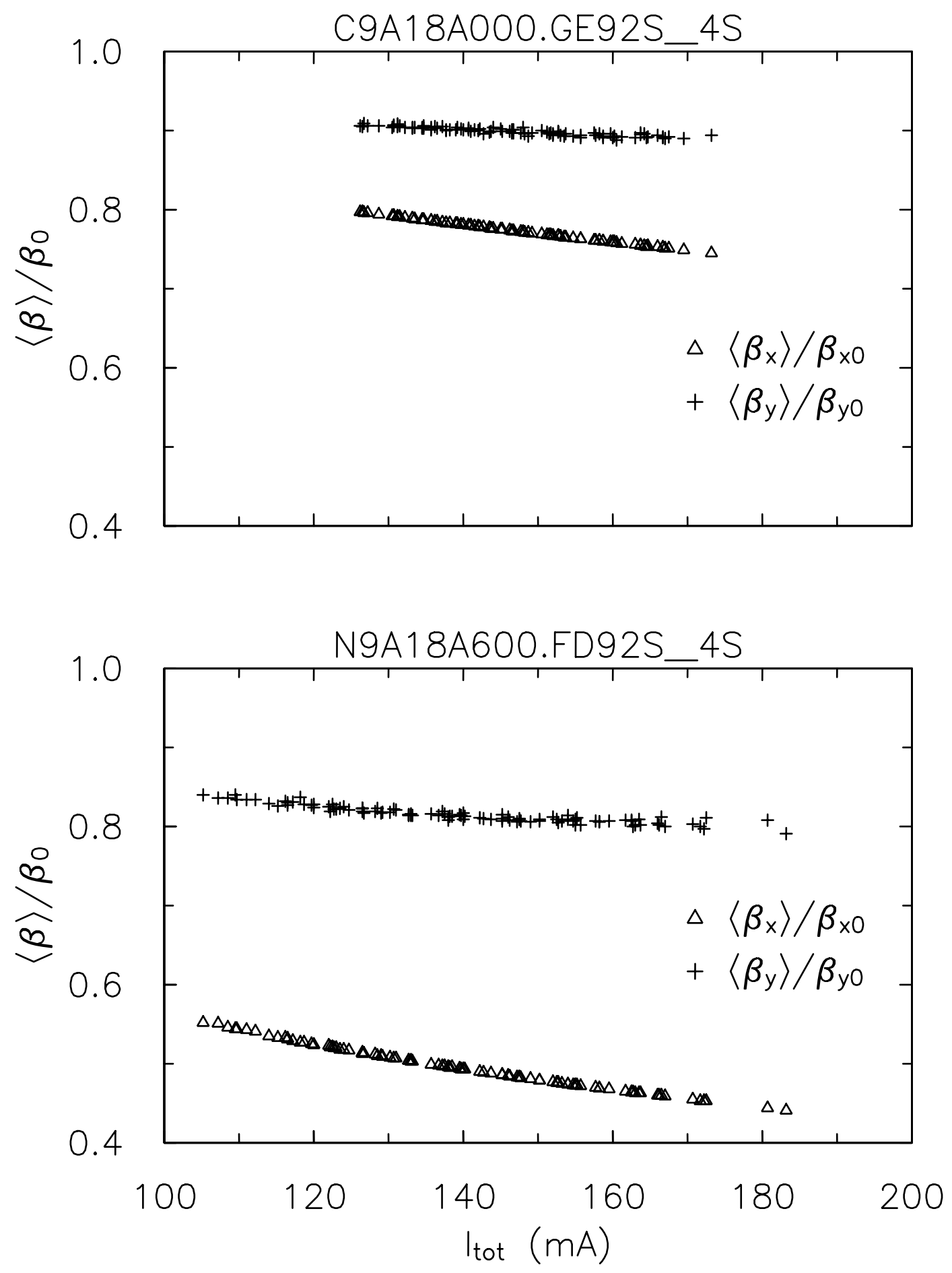

Figure 3: Average beta $\langle\beta\rangle \equiv\left(\beta_{+}+\beta_{-}\right) / 2$ normalized by $\beta_{0}$ as a function of total current. 

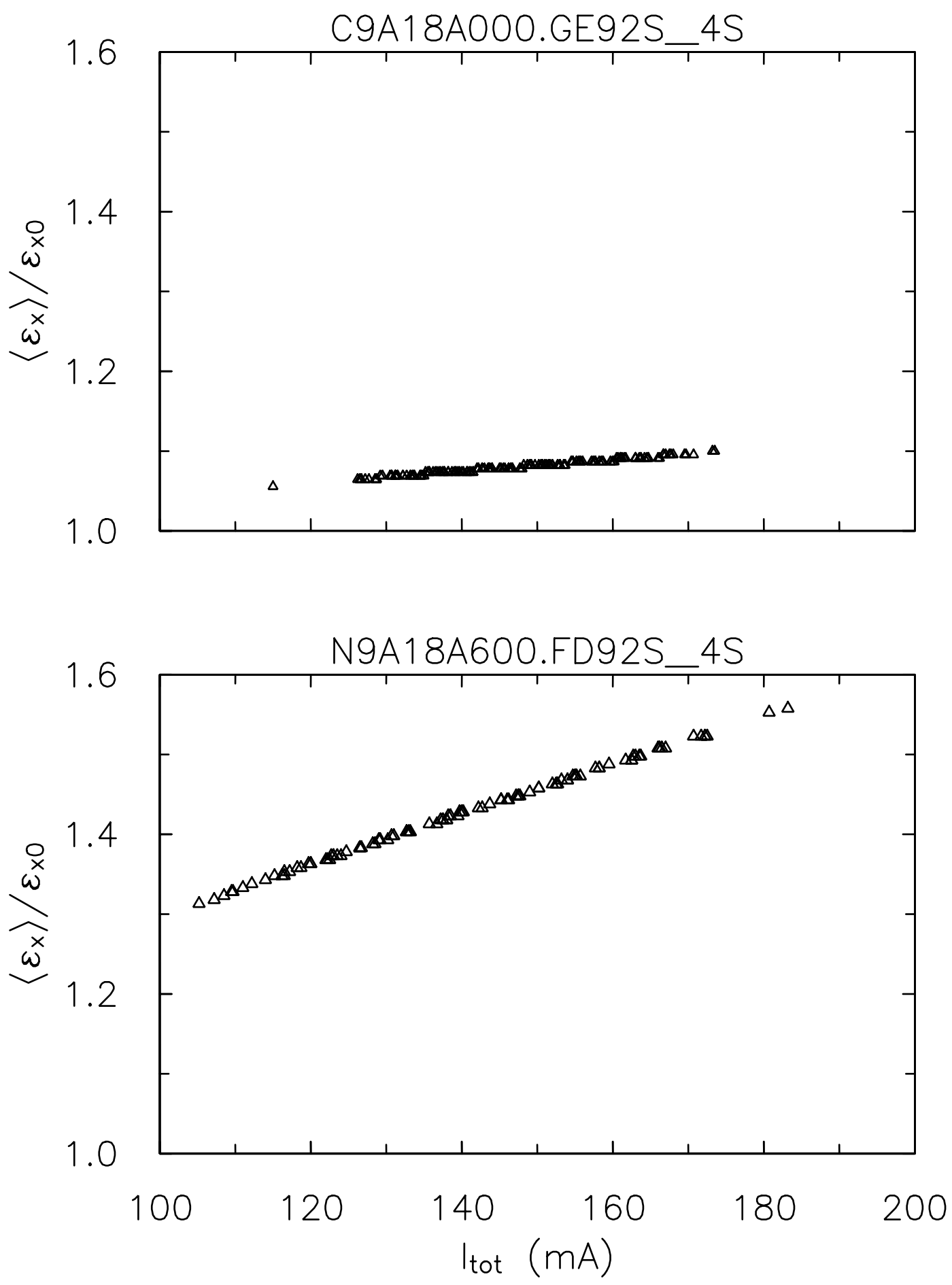

Figure 4: Average horizontal emittance $\left\langle\epsilon_{x}\right\rangle \equiv\left(\epsilon_{x+}+\epsilon_{x-}\right) / 2$ normalized by $\epsilon_{x 0}$ as a function of total current. 


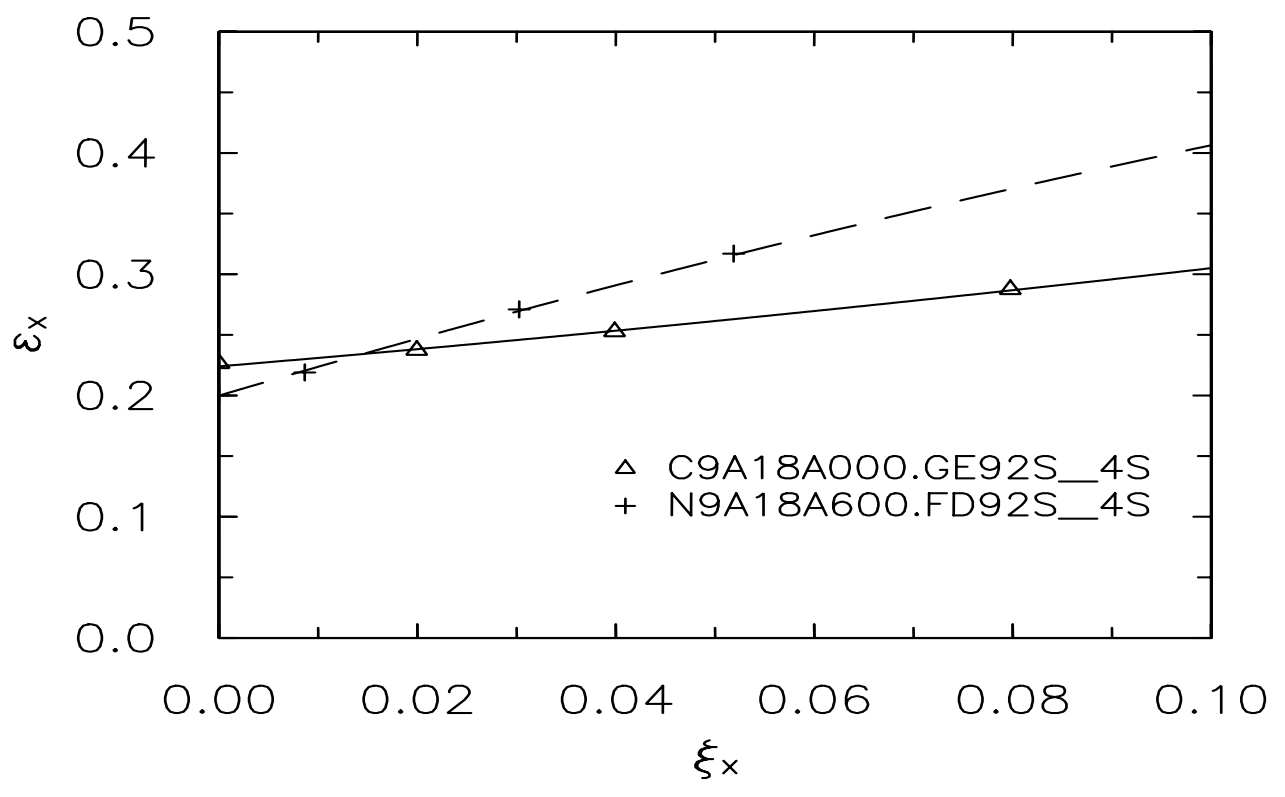

Figure 5: Horizontal emittance as a function of beam-beam parameter $\xi_{x}$ for the N9A18A600.FD92S_4S and C9A18A000.GE92S_4S lattices. 

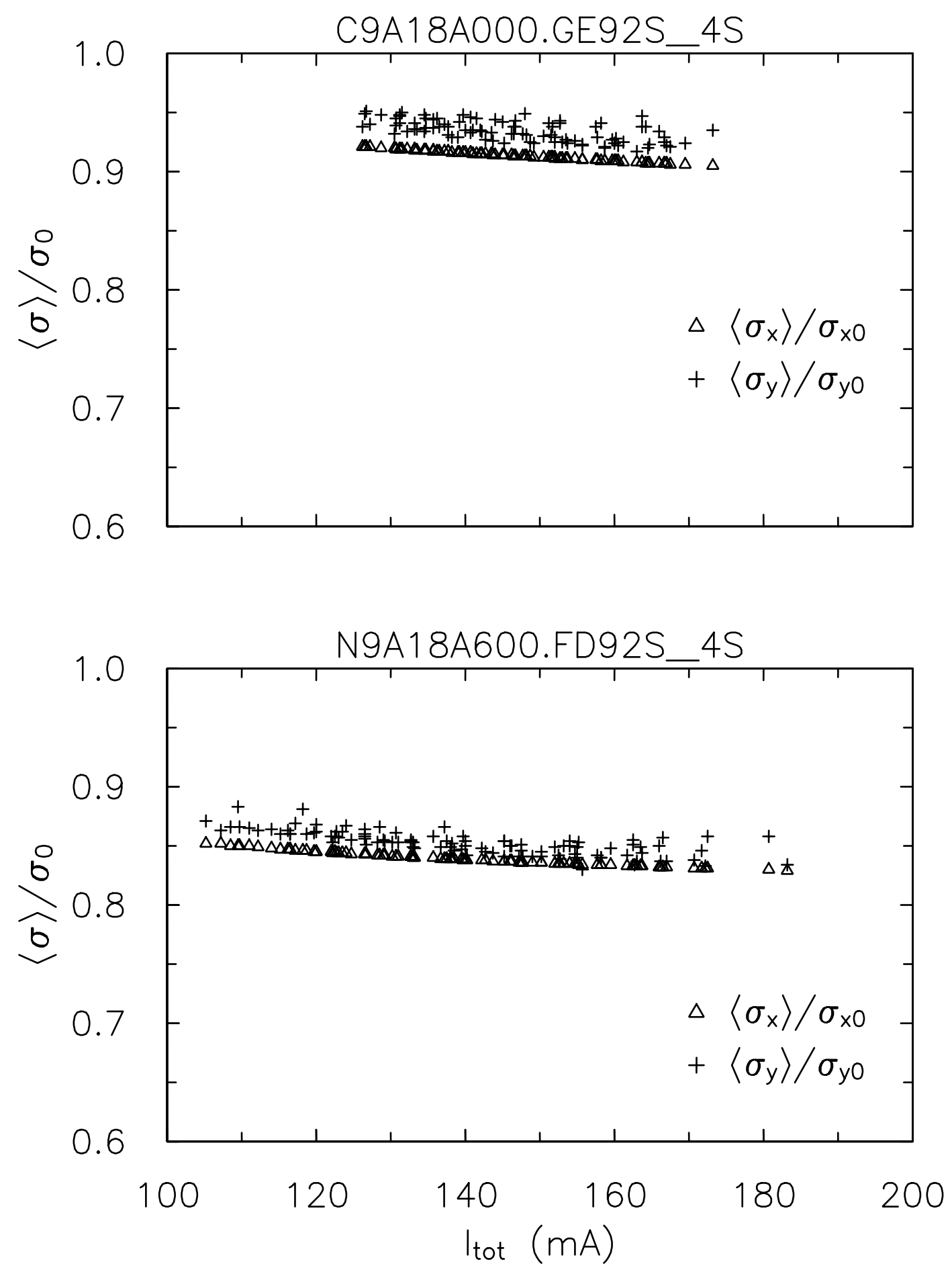

Figure 6: Average beam sigma $\langle\sigma\rangle \equiv\left(\sigma_{+}+\sigma_{-}\right) / 2$ normalized by $\sigma_{0}$ as a function of total current. 

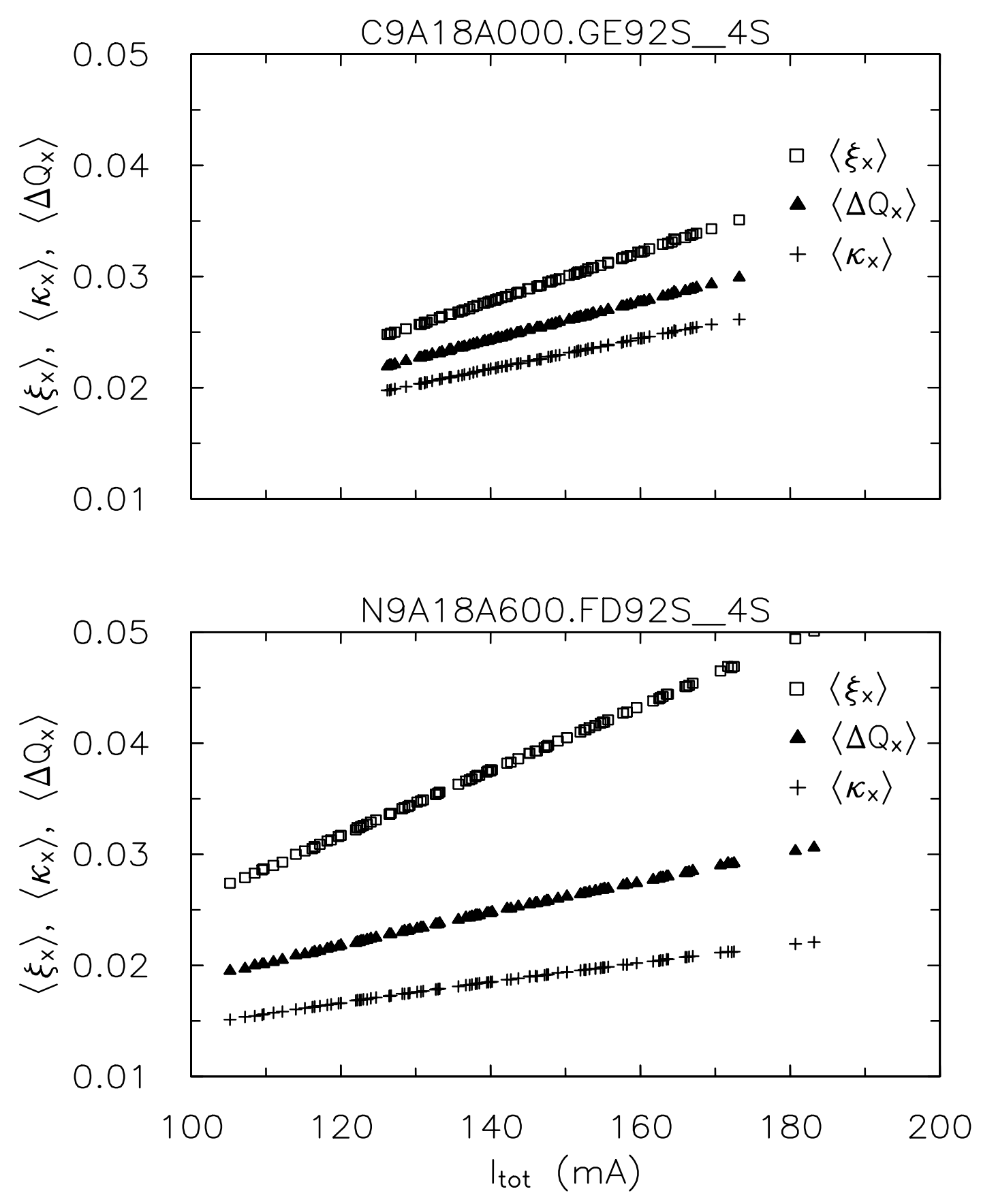

Figure 7: Average $\left\langle\xi_{x}\right\rangle,\left\langle\kappa_{x}\right\rangle$, and $\left\langle\Delta Q_{x}\right\rangle$ as a function of total current. 

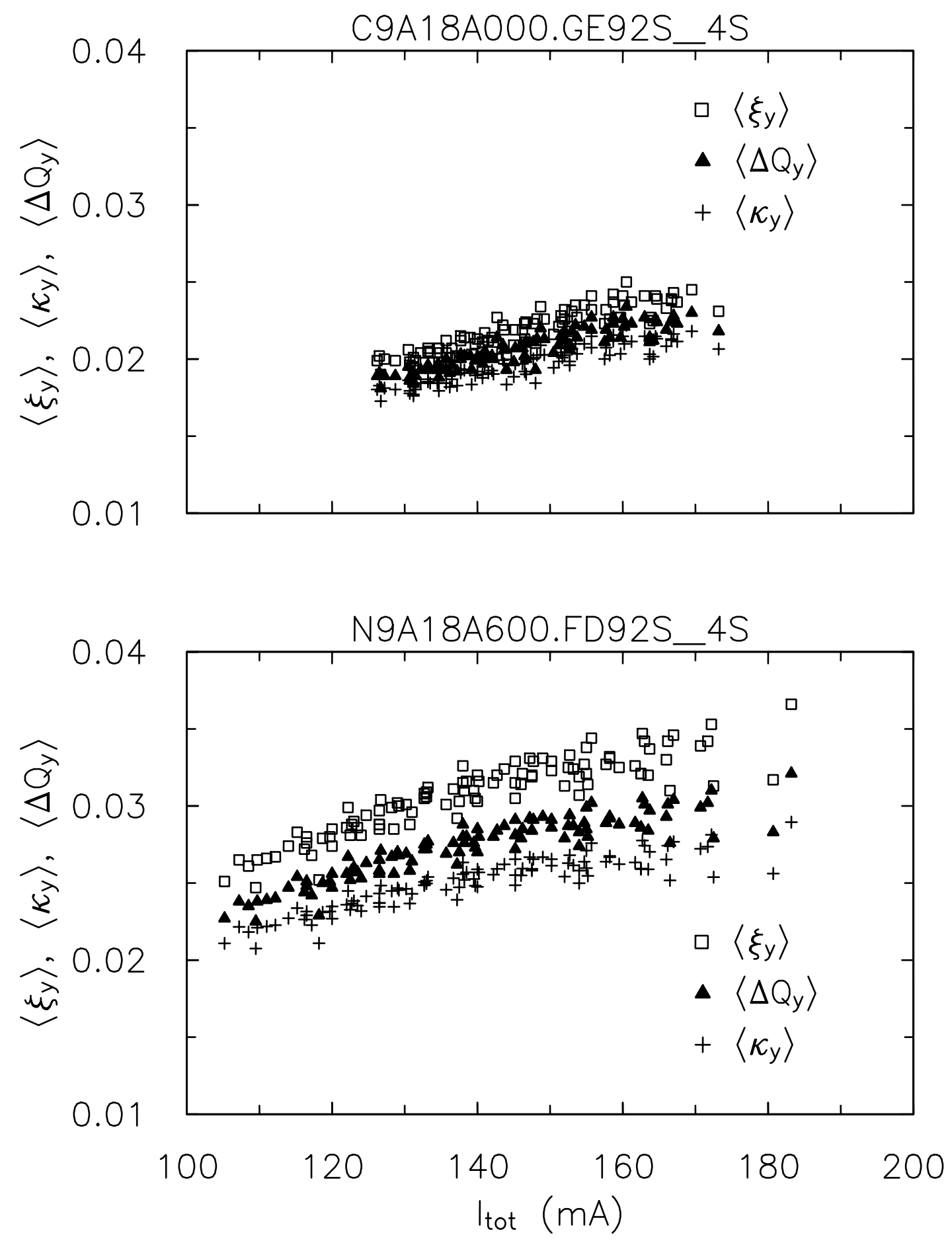

Figure 8: Average $\left\langle\xi_{y}\right\rangle,\left\langle\kappa_{y}\right\rangle$, and $\left\langle\Delta Q_{y}\right\rangle$ as a function of total current. 


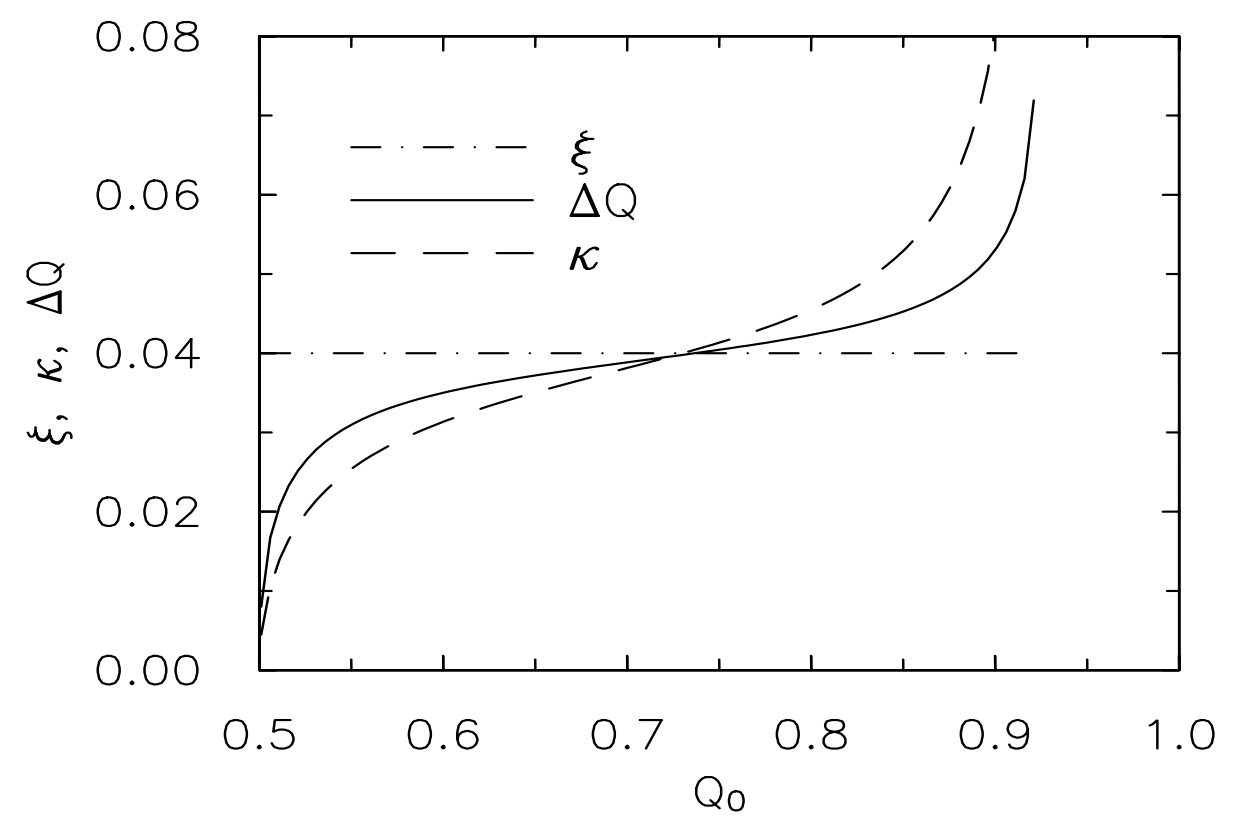

Figure 9: $\xi, \kappa$, and $\Delta Q$ as functions of $Q_{0}$ with $\xi$ somewhat arbitrarily fixed at 0.04 . 


\section{Amplitude dependence}

In terms of single particle dynamics the beam-beam force is nonlinear beyond $1 \sigma$ either horizontally or vertically. The fact that the beam-beam force starts to fall off beyond $1 \sigma$ results in a monotonic decrease of the effective quadrupolar focusing strength with increasing particle oscillation amplitude. This results in the dynamic beta effect being amplitude-dependent with large amplitude particles being relatively unaffected by the dynamic beta effect. This implies that the deleterious effects of reduced single particle lifetime that are associated with a lower $\beta$ (IP) are not present with dynamic beta. In other words, the dynamic beta effect is materially different from using a lattice with a lower $\beta$ (IP).

The amplitude dependence of the dynamic beta effect was explored with a simple 1-dimensional tracking program. The procedure was as follows: The one turn transport map from IP to IP was taken to have the same form as the right hand side of Eq. (1) with the $-1 / 2 f$ kick terms replaced with the full amplitude dependent beambeam kick as given by the complex error function formula of Bassetti and Erskine[5]. Particles were seeded at different amplitudes and tracked for 300 turns. For a single particle the resulting motion in phase space was fitted to an ellipse and a value for $\beta$ extracted. Figure 10 shows the dependence of $\beta / \beta_{0}$ on oscillation amplitude $A$ for both the horizontal and vertical planes. As can be seen, $\beta$ is insensitive to changes in amplitude for the particles with oscillation amplitudes below about $2 \sigma$. This implies

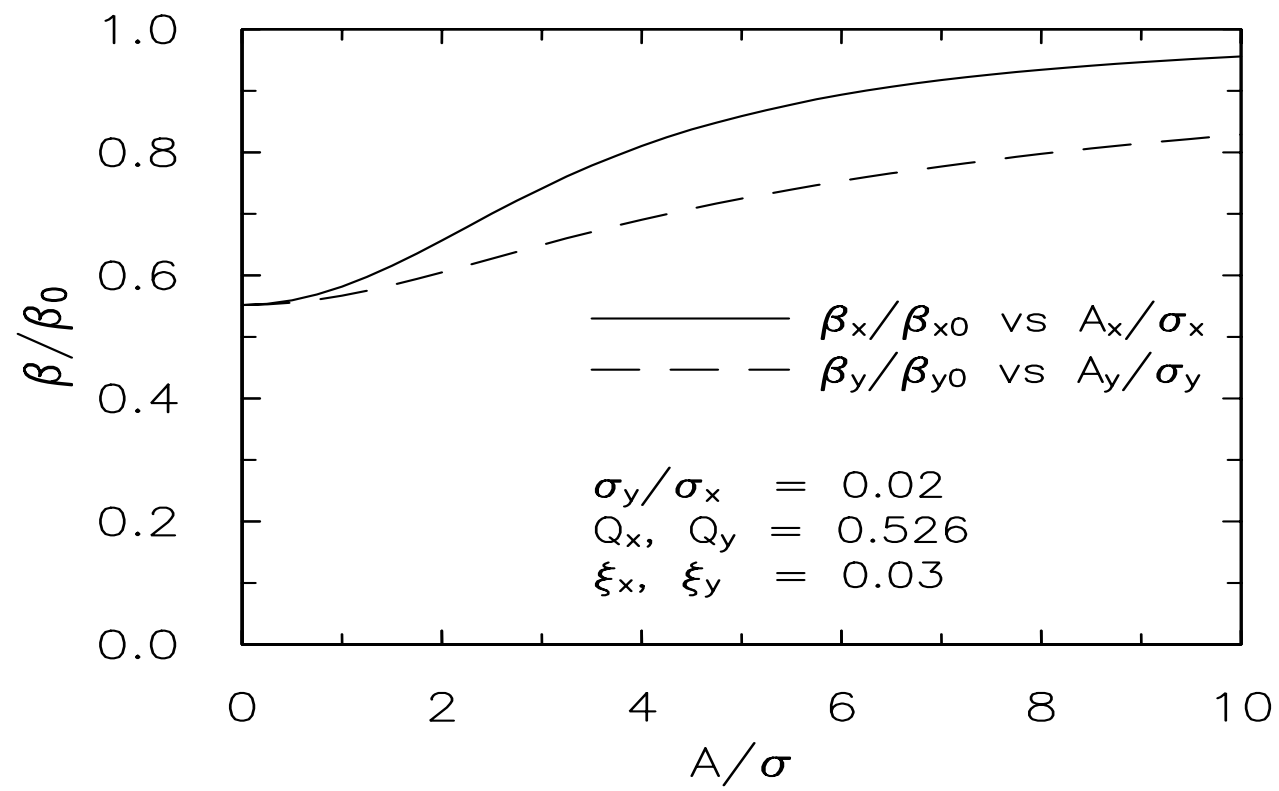

Figure 10: Relative beta as a function of oscillation amplitude for both the horizontal plane (solid line) and the vertical plane (dashed line). 
that the amplitude dependent effects on the luminosity are small. In the tails of the beam, where $A_{x} \gtrsim 10 \sigma_{x}$ or $A_{y} \gtrsim 50 \sigma_{y}$, the dynamic beta effect is seen to be small.

\section{Luminosity Considerations}

From figure 5 it is seen that for the particular lattices considered $\epsilon_{x}$ is an increasing function of $\xi_{x}$. It can be argued that this increase in $\epsilon_{x}$ with increasing $\xi_{x}$ results in an increased $\sigma_{x}$ with an attendant decrease in luminosity. One possibility then for increasing luminosity is to design a lattice in which $\epsilon_{x}$ decreases with increasing $\xi_{x}$. Unfortunately, this might not be practical in CESR. To see this, consider $\mathcal{H}_{x}(s)$ as given by Eq. (17). The dynamic beta effect does not affect $\eta(s)$ or the damping partition numbers since the IP is in a dispersion free zone. The effect of dynamic beta upon $\epsilon_{x}$ is thus through the beta-wave that is generated. In order to make $\epsilon_{x}$ be a decreasing function of $\xi_{x}$ one would have to change where in the lattice $\beta_{x}$ is increased and where it is decreased. From Eq. (15), since $\mu_{0}$ is confined to be near a half-integer (we want to have a significant dynamic beta), the only way to shift the beta-wave is to shift around the phase advance $\phi_{x 0}(s)$. To shift $\phi_{x 0}(s)$, however, would change where the pretzel maxima are. It is therefore not clear (at least not at this point in time) whether a decreasing $\epsilon_{x}$ is compatible with multibunch operation.

Another tactic for increasing $\mathcal{L}$ might be to run with $\mu_{y}$ nearer to the half-integer instead of $\mu_{x}$. The reason for this is contained in the equation

$$
\mathcal{L}=\frac{I \gamma \xi_{y}(1+r)}{2 e r_{e} \beta_{y 0}}
$$

where $r=\sigma_{y} / \sigma_{x}$. Using the dynamic beta effect it could be argued that one should be able to increase $\xi_{y}$ above the traditional limit in CESR of 0.03 to 0.04 . The problem is that the hourglass effect[6] is non-negligible in CESR so with $\beta_{y}$ comparable to $\sigma_{z}$ it is not clear that using the dynamic beta effect to increase $\xi_{y}$ and lower $\beta_{y}$ is helpful to the luminosity without simultaneously lowering $\sigma_{z}$. Additionally, it is not clear that $\Delta Q_{y}$ is the limiting factor with the current conditions. This is especially true since the crossing angle increases the horizontal resonance strengths faster than the vertical resonance strengths. Experimentally it was found awhile back that switching to a lattice where the vertical tune was closer to the half-integer made things worse. Operationally, however, conditions have changed since that experiment (for one we now have a crossing angle) and therefore, like the stock market, past experience is not necessarily indicative of future performance.

Simulations are one possible way to try to understand how having $\mu_{y}$ near the halfinteger will affect luminosity. Unfortunately, the beta-wave generated by the dynamic beta effect may affect coupling compensation, etc. Ultimately machine studies will have to be done if an answer is to be obtained. 


\section{Acknowledgements}

My thanks to Jim Welch, Dave Rubin, and Dave Rice for useful discussions. My thanks also to Dave Rubin for helping with the emittance calculation.

\section{References}

[1] Alexander Chao, "Beam-Beam Instability," from Physics of High Energy Particle Accelerators, p. 201, AIP Conference Proceedings No. 127, Melvin Month et. al, eds. (1985).

[2] C. Bovet, R. Gouiran, I. Gumowski, and K. H. Reich, "A Selection of Formulae and Data Useful for the Design of A. G. Synchrotrons," CERN/MPS-SI/Int. DL/70/4 (1970).

[3] Matthew Sands, The Physics of Electron Storage Rings, An Introduction. SLAC121 Addendum (1970).

[4] J. Seeman, "Observations of the Beam-Beam Interaction," Proc. Joint US-CERN school on Part. Acc., Nonlinear Dynamics Aspects of Part. Acc, Sardinia, 121, (1985).

[5] M. Bassetti and G. Erskine, CERN-ISR-TH/80-06 (1970).

[6] Stephen Milton, "Calculation of How the Ratio $\beta^{*} / \sigma_{z}$ Affects the Maximum Luminosity Obtainable: The HourGlass Effect," Cornell CBN 89-1 (1989). 